Diabetologe 2010 - 6:169

DOI 10.1007/s11428-010-0554-2

(c) Springer-Verlag 2010

\author{
W. Rathmann \\ Institut für Biometrie und Epidemiologie, Deutsches Diabetes-Zentrum, \\ Leibniz-Zentrum für Diabetes-Forschung, Düsseldorf
}

\title{
Epidemiologie des Diabetes
}

„Epidemiology provides a scientific basis for clinical and public health practice. Indeed, epidemiology can be used to guide how we define, diagnose, and screen for diabetes, to describe the present and future burden of diabetes, and to highlight opportunities for intervention."

(William H. Herman, Kelly West Award Lecture 2006)

Anhand epidemiologischer Daten werden diagnostische Kriterien festgelegt, Maßnahmen zur Früherkennung und Prävention des Diabetes entwickelt, genetische und nichtgenetische Risikofaktoren für den Diabetes aufgedeckt sowie Interventionen zur Prävention des Diabetes untersucht. Die Epidemiologie liefert damit einen wertvollen Beitrag neben der Grundlagen- und klinischen Forschung.

Der Typ-2-Diabetes wurde in der Vergangenheit nur als einer von vielen Risikofaktoren der koronaren Herzkrankheit wahrgenommen. Die eigenständige Bedeutung sowohl für die klinische Behandlung als auch für die Versorgung aus $\mathrm{Pu}$ blic-Health-Perspektive hat jedoch deutlich zugenommen. Dies liegt u. a. an der Zunahme der weltweiten Diabetesprävalenz. Die „International Diabetes Federation" schätzt, dass sich die weltweite Zahl der Menschen mit Diabetes von derzeit 285 Mio. in den nächsten 20 Jahren auf 438 Mio. erhöhen wird. Schätzungsweise $70 \%$ davon werden in Ländern mit niedrigen oder mittleren durchschnittlichen Einkommen leben („Entwicklungs- oder Schwellenländer“). Der Typ-2-Diabetes stellt aufgrund der hohen Prävalenz und der erhöhten medizinischen Inanspruchnahme aber auch eine Belastung für die Finanzierung der Gesundheitssysteme in ökonomisch starken Ländern dar.

\section{$\checkmark$ Die Epidemiologie liefert wichtige Erkenntnisse zur Diagnostik und Prävention des Diabetes}

In den folgenden Artikeln wird ein kurzer Überblick über aktuelle Studien zur Diabetesepidemiologie in Deutschland gegeben. Im ersten Beitrag werden Ergebnisse zu Prävalenz und Inzidenz des Typ-2-Diabetes sowie zur Häufigkeit des „Prädiabetes" in der Region Augsburg zusammengefasst (Rathmann u. Meisinger). Seit Mitte der 1980er Jahre wurden in Augsburg mehrere bevölkerungsbasierte Studien (MONICA, KORA) durchgeführt. In diesen Studien ergab sich z. B. eine Prävalenz des unentdeckten Diabetes, die etwa so hoch war wie die Häufigkeit des bekannten Diabetes. Im zweiten Artikel fassen Rosenbauer u. Stahl aktuelle Daten zur Häufigkeit des Typ-1- und Typ2-Diabetes im Kindes- und Jugendalter in Deutschland vor dem Hintergrund internationaler Daten zusammen. Trotz der Zunahme von Übergewicht und Adipositas in dieser Altersgruppe ist bisher keine Zunahme des Typ-2-Diabetes festzustellen. Der dritte Beitrag von A. Icks behandelt die aktuelle Inzidenz schwerwiegender gesundheitlicher Ereignisse wie Amputationen, Erblindungen, terminale Niereninsuffizienz und kardiovaskuläre Erkrankungen bei Personen mit Diabetes im Vergleich zur nichtdiabetischen Population sowie Veränderungen im Sinne der so genannten „St.-Vincent-Ziele“. Im folgenden Artikel von Kowall u. Mielck wird der Zusammenhang von sozialer Lage und Typ-1- und Typ-2-Diabetes behandelt („Trifft es Arme öfter?“). Der Einfluss der sozialen Lage auf die Gesundheit macht auch bei Patienten mit manifestem Diabetes nicht Halt, so leiden Diabetespatienten mit niedrigem sozialem Status häufiger an Komplikationen. Im letzten Betrag fasst C. Herder die Ergebnisse genetisch-epidemiologischer Studien zusammen. Bislang sind etwa 25 Risikogene des Typ-2-Diabetes bekannt, die allerdings nur 5-10\% der genetischen Prädisposition in der Bevölkerung erklären. Die kommerzielle Vermarktung von entsprechenden Gentests ist daher definitiv verfrüht, da zurzeit einfache Größen wie Alter, Adipositas und positive Familienanamnese eine weitaus bessere Abschätzung des Diabetesrisikos erlauben.

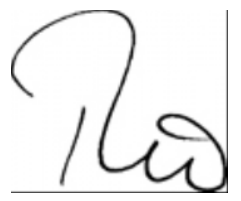

Wolfgang Rathmann

\section{Korrespondenzadresse Dr.W. Rathmann}

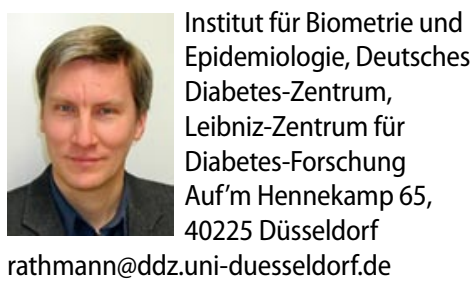

